\title{
Impact of interface thermodynamics on Al-induced crystallization of amorphous SixGe1-x alloys - CORRIGENDUM
}

Christian A. Niedermeier, Zumin Wang, and Eric J. Mittemeijer

doi: 10.1557/jmr.2014.49, Published by Materials Research Society with Cambridge University Press, 7 March 2014.

In Niedermeier et al. ${ }^{1}$, page 2 , second column, line 45: a- $\mathrm{Si}_{0.41} \mathrm{Ge}_{0.61}$ should read a-Si $\mathrm{Si}_{0.41} \mathrm{Ge}_{0.59}$.

The authors regret the mistake.

\section{REFERENCE}

1. C.A. Niedermeier, Z. Wang, E.J. Mittemeijer: Impact of interface thermodynamics on Al-induced crystallization of amorphous SixGe1-X alloysnanoparticles. J. Mater. Res. 29(6), 786-792 (2014). 